\title{
BMC Dermatology reviewer acknowledgement, 2012
}

Hayley Henderson

\section{Contributing reviewers}

The editors of BMC Dermatology would like to thank all our reviewers who have contributed to the journal in Volume 12 (2012).

Ole Ahlehoff

Denmark

Ayadi Ali

Tunisia

Marco Calogero Amato

Italy

Giuseppe Argenziano

Italy

Benoît Arveiler

France

Andrzej Bieniek

Poland

Ivan Birch

United Kingdom

Anthony Bullock

United Kingdom

Ian Burgess

United Kingdom

John Burgess

Australia

Ali Cadili

Canada

Sergio Chimenti

Italy

Tadd Clayton

New Zealand

\author{
Arnon Cohen \\ Israel \\ Darren Day \\ New Zealand \\ Marília-Gerhardt De Oliveira \\ Brazil
}

Esther De Vries

Netherlands

Chukwuma Ekpebegh

South Africa

Nadia El Fekih

Tunisia

Brigitte Essers

Netherlands

John Foerster

United Kingdom

Asli Gelincik

Turkey

Anthony Goon

Singapore

Shoshana Greenberger

Israel

Metin Gungormus

Turkey

Melvin Hayden

USA

\author{
Masaru Honma \\ Japan \\ Nicholas Inston \\ United Kingdom \\ Slavenka Jankovic \\ Serbia \\ Jams Jayatilake \\ Sri Lanka \\ Myungshin Kim \\ Korea South \\ Jacob Levitt \\ USA \\ Richard Liebano \\ Brazil \\ Marie Loden \\ Sweden \\ Blazej Meczekalski \\ Poland \\ Kim Meeuwis \\ Netherlands \\ Elvira Moscarella \\ Italy \\ Luigi Naldi \\ Italy \\ Lill Tove Nilsen \\ Norway
}

Correspondence: hayley.henderson@biomedcentral.com

BioMed Central, Floor 6, 236 Gray's Inn Road, London WC1X 8HB, United

Kingdom

\section{() Biomed Central}

(c) 2013 Henderson; licensee BioMed Central Ltd. This is an Open Access article distributed under the terms of the Creative Commons Attribution License (http://creativecommons.org/licenses/by/2.0), which permits unrestricted use, distribution, and reproduction in any medium, provided the original work is properly cited. 
Emeka Nweze

Nigeria

Monia Orciani

Italy

Sean Palecek

USA

Aldona Pietrzak

Poland

Olivier Piot

France

Rosa Maria Ponce

Mexico
Toshiaki Saida

Japan

Omar Sedrati

Morocco

Tor Shwayder

USA

Georgios Stamatas

France

Denise Steiner

Brazil

Joanna Stewart

New Zealand
Kursad Unluhizarci

Turkey

V Vijayanath

India

Sümer Yamaner

Turkey

doi:10.1186/1471-5945-13-4

Cite this article as: Henderson: BMC

Dermatology reviewer acknowledgement,

2012. BMC Dermatology 2013 13:4. 